\title{
A NEW SURVIVABILITY MEASURE FOR MILITARY COMMUNICATION NETWORKS
}

\author{
Haizhuang Kang \\ Clive Butler \\ Qingping Yang \\ Department of Manufacturing and Engineering Systems \\ Brunel University, Middlesex, UB8 3PH, UK \\ Jiamo Chen \\ Department of Information Engineering \\ XIDIAN University, Xi' an, 710071, P R China
}

\begin{abstract}
A new measure for survivability of military communication networks based upon topological structures is presented in this paper. The proposed measure can be used to evaluate and enhance the survivability of military communication networks, which is illustrated through case studies. The computer simulation results have shown that the new measure can well reflect the survivability of networks. It can be used as a reliable criterion for estimating the survivability of networks and designing networks with high survivability.
\end{abstract}

\section{INTRODUCTION}

The measurement of survivability of military communication networks (MCNs) has been studied more than ten years. Many different measures have been reported. Most existing survivability measures are based upon graph theory. In the earlier, it was quite simple by using either node connectivity or minimum cut tree. It has been increasingly realized that the measurement for survivability of MCNs is a complicated problem because the network is subject to deliberate and/or random attack. Since the destruction of a network is essentially a process, in which the nodes of the network are gradually destroyed until being completely disconnected, a survivability measure should be based upon such a process and its definition should reflect the survivability of the network during the entire destruction process. As the network may be damaged in many different ways, survivability measure can also be defined based upon different processes. The minimum cut tree is used in reference [2], but it results in complicated computations. In this paper, a new survivability measure for $\mathrm{MCNs}$ has been proposed to enhance accuracy and efficiency of survivability measure.

\section{SURVIVABILITY OF MCNs}

The survivability of an MCN is its ability to maintain communication among the nodes when it is subject to deliberate destructions. The proposed survivability measure made the following assumption:

(1) The node of the network has only two status, damaged or undamaged.

(2) The link between two nodes is wireless ( because most military communication networks are formed based upon mobile radio communication). Therefore the link damage is not taken into account.

(3) Only one node is destroyed or removed every time, and this node is the most important one in the network, which means the worst case occurs every time.

The new measure for the survivability of MCNs is based upon the estimation of network connectivity in a complete destruction process, i.e. the network connectivity is summed with the node of network being removed one by one until the network becomes disconnected.

Assuming the number of nodes in a given $\mathrm{MCN} G$ is $n$, the survivability measure (SM) for the network is defined as:

$$
S M(G)=\sum_{k=0}^{m-1} C M(k)
$$

where $\mathrm{CM}(0)$ is the connectivity measure of network $\mathrm{G}$; $\mathrm{CM}(\mathrm{k})$ is the connectivity measure of network $\mathrm{G}_{k}$ which was produced by removing the most important node from the network $G_{k-1}, k=1,2, \ldots, m-1 ; m$ is the number of the nodes which have to be removed before the network becomes totally disconnect (i.e. all the rest nodes are isolated).

The definition of $\mathrm{CM}(\mathrm{k})$ is given by

$$
C M(k)=\sum_{i=1}^{n-k-1} \sum_{j=i+1}^{n-k} N C_{k}(i, j)
$$


here $\mathrm{NC}_{k}(i, j)$ is node connectivity between $i$ and $j$ in the network $G_{k} ; n-k$ is the number of nodes in network $G_{k}$.

The node connectivity $\mathrm{NC}_{\mathrm{k}}(\mathrm{i}, \mathrm{j})$ is defined as

$$
N C_{k}(i, j)=\sum_{t=1}^{x} \frac{1}{J N(t)}
$$

Where $x$ is the number of independent paths between nodes $\mathrm{i}$ and $\mathrm{j}$ (independent paths means that there is no common node between them); $\mathrm{NN}(\mathrm{t})$ is the number of jumps along the $t$-th independent path between nodes $i$ and $\mathrm{j}$.

For example, the network $G_{k}$ shown in Fig. 1 has three independent paths between nodes $\mathrm{i}$ and $\mathrm{j}$. Thus, we have $x=3$ :

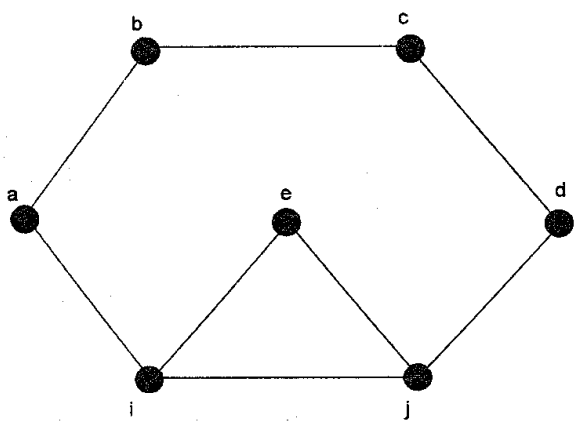

Fig. 1 network $G_{k}$

$$
\begin{array}{ll}
\text { path } 1: \mathrm{i}-\mathrm{j} & \mathrm{NN}(1)=1 ; \\
\text { path } 2: \mathrm{i}-\mathrm{e}-\mathrm{j} & \mathrm{JN}(2)=2 ; \\
\text { path } 3: \mathrm{i}-\mathrm{a}-\mathrm{b}-\mathrm{c}-\mathrm{d}-\mathrm{j} & \mathrm{NN}(3)=5
\end{array}
$$

$$
N c_{k}(i, j)=1+1 / 2+1 / 5
$$

The node connectivity (NC) between nodes $i$ and $j$ depends not only on the number of the independent paths between them, but also on the jumps of the path. If there is a direct link between nodes $\mathrm{i}$ and $\mathrm{j}$ (as path 1 ), its contribution to $\mathrm{NC}_{\mathrm{k}}(\mathrm{i}, \mathrm{j})$ is 1 , it also means that the survivability of this link is $100 \%$, i.e. it is assumed that the link cannot be damaged. On the other hand, the contribution of the path 2 or the path 3 to $\mathrm{NC}_{\mathrm{k}}(\mathrm{i}, \mathrm{j})$ is less than 1 , because it is possible to be destroyed. The more the jumps of the path, the less the contribution to the $\mathrm{NC}_{\mathrm{k}}(\mathrm{i}, \mathrm{j})$.

The importance of node i, F(i) is defined as the number of all independent paths including the node $i$ in the network $\mathrm{G}_{\mathrm{k}}$, where $\mathrm{i}=1,2, \ldots \mathrm{n}-\mathrm{k}$.

It should be noted that if there are two paths which share common nodes, the path which have minimum jumps should be chosen.
CASE STUDY

Two networks $G$ and $G_{1}$ are shown in Fig. 2 and 3.

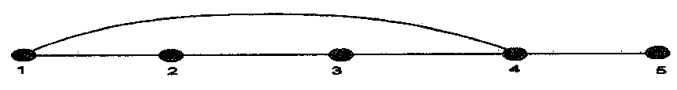

\begin{tabular}{|c|c|c|}
\hline $\mathrm{k}=0$, & $\begin{array}{l}\mathrm{NC}_{0}(1,2)=1+1 / 3 \\
\mathrm{NC}_{0}(1,4)=1+1 / 3\end{array}$ & $\begin{array}{l}\mathrm{NC}_{0}(1,3)=1 / 2+1 / 2 \\
\mathrm{NC}_{0}(1,5)=1 / 2\end{array}$ \\
\hline & $\begin{array}{l}\mathrm{NC}_{0}(2,3)=1+1 / 3 \\
\mathrm{NC}_{0}(2,5)=1 / 3\end{array}$ & $\mathrm{NC}_{0}(2,4)=1 / 2+1 / 2$ \\
\hline & $\begin{array}{l}\mathrm{NC}_{0}(3,4)=1+1 / 3 \\
\mathrm{NC}_{0}(4,5)=1\end{array}$ & $\mathrm{NC}_{0}(3,5)=1 / 2$ \\
\hline
\end{tabular}

Fig. 2 Network G

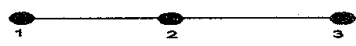

Fig. 3 Network $\mathrm{G}_{1}$

In the network $\mathrm{G}$,

Thus

$$
C M(0)=\sum_{i=1}^{4} \sum_{j=i+1}^{5} N C_{0}(i, j)=9.67
$$

The importance of each node in the network $G$ is as follows:

$F[1]=11 ; \quad F[2]=10 ; \quad F[3]=11 ; \quad F[4]=13 ; \quad F[5]=4$.

The destruction of the most important node 4 leads to network $\mathrm{G}_{1}$ as shown in Fig. 3.

In the network $G_{1}$ :

$\mathrm{k}=1, \quad \mathrm{NC}_{1}(1,2)=1 \quad \mathrm{NC}_{1}(1,3)=1 / 2$ $\mathrm{NC}_{1}(2,3)=1$

Thus,

$$
C M(1)=1+\frac{1}{2}+1=2.5
$$

The importance of each node in the network $G_{1}$ is given by:

$F[1]=2 ; \quad F[2]=3 ; \quad F[3]=2 ; \quad F[4]=0 ; \quad F[5]=0$.

If the most important node 2 is removed, the network will become completely isolated. Therefore, $\mathrm{m}=2$ and $\mathrm{SM}=\mathrm{CM}(0)+\mathrm{CM}(1)=9.67+2.5=12.17$ 


\section{THE CHARACTERISTIC OF THE NEW MEASURE}

- $\quad$ There are many ways to decompose a network. For a grid network with 20 nodes, the number of possible decomposed paths may amourt to 200 thousand. Using the proposed measure, the decomposition process has become much simpler because it is only necessary to remove the most important node in the network every time. Significant time saving can be achieved in computing the new measure. Of course, this assumes the worst case every time a node is destroyed, and the proposed survivability measure is thus the minimum value of survivability for this network.

The new survivability measure has combined both node connectivity factor and link connectivity factor, and has thus provided a unique criterion for estimating the survivability of network. The new measure is more reliable and efficient than most existing ones. The importance of nodes $F(i)$ can be obtained with the result of SM and no other parameters are required.

- In order to verify the proposed survivability measure, a test is conducted using computer software simulation. During the test, the network is subject to random destruction until $50 \%$ nodes of the network have been damaged (removed). The test is repeated for 10,000 times, the probability that the rest nodes are still in connectivity is calculated. The larger this probability, the higher the survivability of the network. Here are four networks which have same number of nodes and links but different configurations.

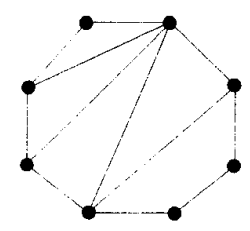

Fig.4

$\mathrm{SM}=53.73$

Probability $=38.74 \%$

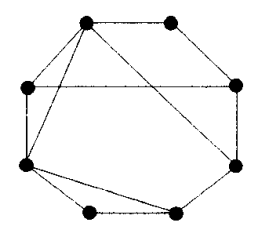

Fig.5

$$
\mathrm{SM}=63.75
$$

Probability $=47.58 \%$

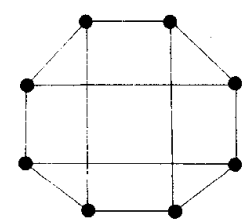

Fig. 6

$$
\mathrm{SM}=79.75
$$

Probability $=38.74 \%$

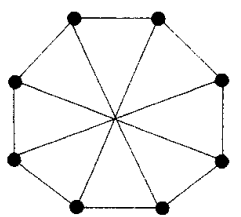

Fig. 7
The results of computer simulation show that the more the SM, the more the probability of survivability of network. So this measure is credible.

\section{THE RESULT OF SM FOR STANDARD GRID NETWORK}

A software for calculating the SM has been developed. With standard grid networks (Fig.8) within 35 nodes, the

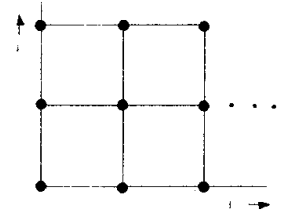

Fig. 8 network nodes $=\mathrm{i} \times \mathrm{j}$ value of $\mathrm{SM}$ and the computation time are shown in Fig. 9 and 10 . The computation time has been significantly reduced, compared with [2]. So the new measure is practical for estimating the survivability of network in real time.

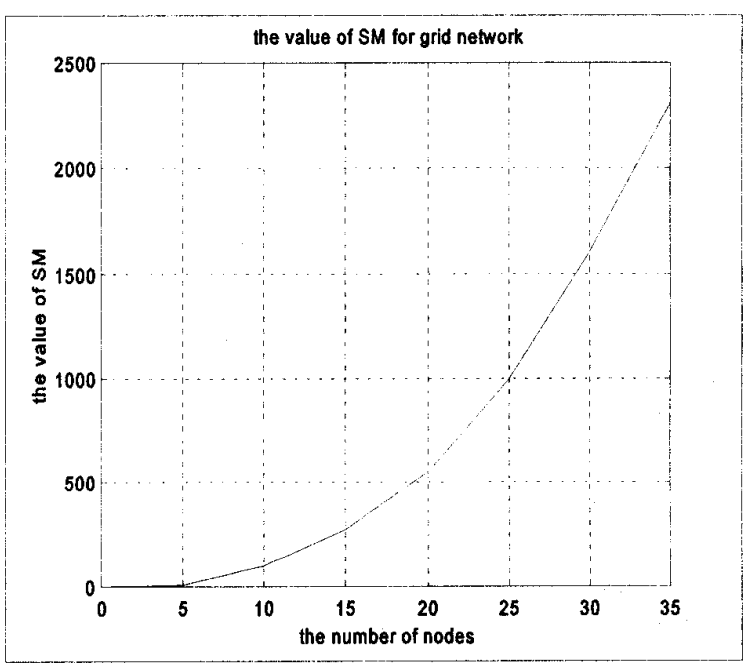

Fig. 9 


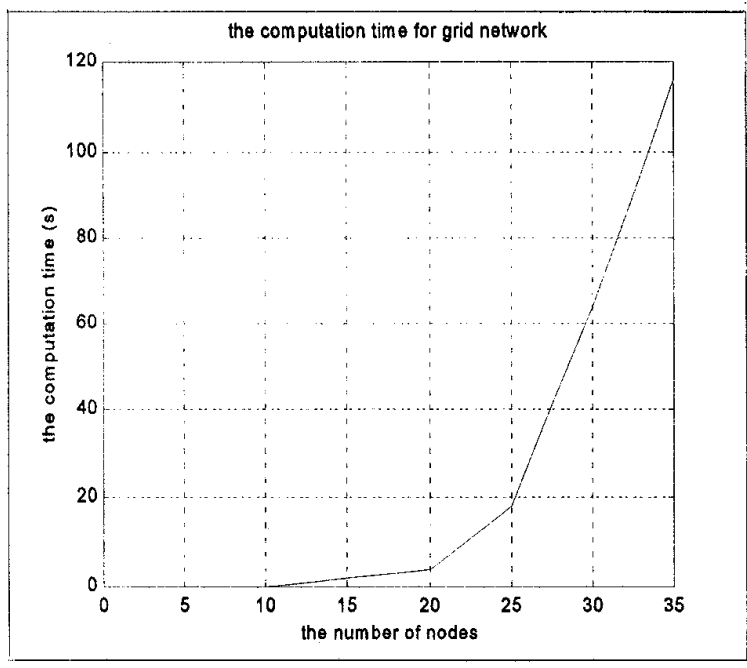

Fig. 10

\section{OPTIMIZATION OF NETWORKS USING THE CRITERIA OF SM}

The SM has provided a quick and efficient method for evaluating the survivability of the network. It can also be used to optimize the network to enhance the survivability of the network. One example is given here, which relies upon adjusting the links of network to enhance the survivability of the network.

It can be seen from the above discussions that a network with a higher survivability tends to be more balanced, which means that the importance of the network nodes tend to be more uniform, ideally equal. In the light of this criterion, the process for optimization of the network can be formulated as follows:

(1) Calculate the SM of the initial network;

(2) Find the two nodes $I$ and $j$ with the maximum $\mathrm{NC}_{\mathrm{k}}(\mathrm{i}, \mathrm{j})$ between them, and remove the link between them;

(3) Fix a link between the two nodes of the least importance;

(4) Repeat the steps 1-3 until the SM value stops increasing.

A network (fig.11) is chosen from [2] for illustrating this process.

The SM for the initial network (fig.11) is $\mathrm{SM}=60.67$, with the importance of eight nodes given by $F[1$ to $8]=[15,38,28,39,21,31,29,24]$. Then the network connectivity can be found, e.g.

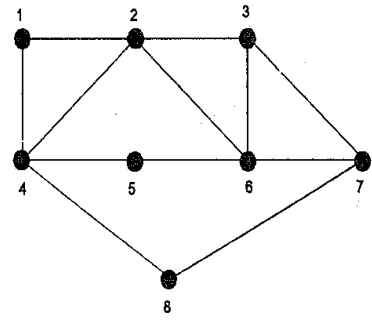

Fig. 11

$$
\begin{aligned}
& \mathrm{NC}_{0}(2,4)=1+1 / 2+1 / 3+1 / 4 \\
& \mathrm{NC}_{0}(2,6)=1+1 / 2+1 / 3 \\
& \mathrm{NC}_{0}(3,6)=1+1 / 2+1 / 2
\end{aligned}
$$

It can be shown that $\mathrm{NC}_{0}(2,4)$ has the maximum value and hence the link between nodes 2 and 4 is removed (Fig. 11). Also as nodes 1 and 5 are least important, a link is created between them, as shown in Fig. 12.

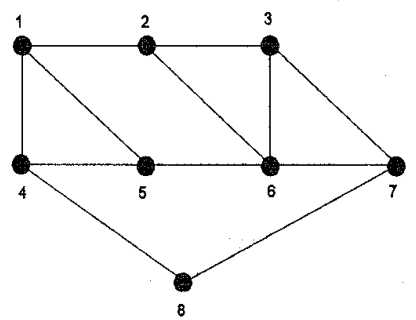

Fig. 12

Now the SM becomes SM=70.53 (Fig.12), and the importance of the eight nodes $F[1$ to 8$]=[35,35,32,39$, $34,37,39,32]$, with the network connectivity:

$$
\begin{aligned}
& \mathrm{NC}_{1}(3,6)=1+1 / 2+1 / 2 ; \\
& \mathrm{NC}_{1}(1,5)=1+1 / 2+1 / 3 ; \\
& \mathrm{NC}_{1}(2,6)=1+1 / 2+1 / 2 ;
\end{aligned}
$$

According to the same criterion, the link between nodes 3 and 6 should be removed and a link between nodes 3 and 8 is added (Fig. 13).

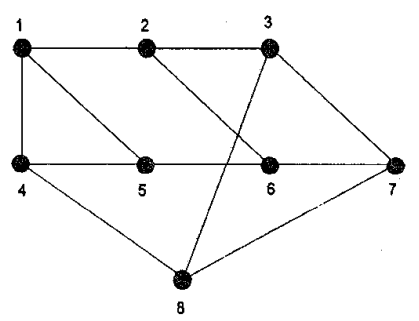

Fig. 13

The network in Fig. 13 has an $\mathrm{SM}=76.17$, and $\mathrm{F}[1$ to $8]=[37,40,37,40,37,40,37,40]$. It can be verified that the network is balanced now because any adjusting will 
not increase the SM value. Therefore no more adjustment is required and the network has an optimised survivability. This result is the same as that given in reference [2].

\section{CONCLUSION}

Nowadays it has come to light that the design for MCNs is a complicated problem. the survivability is becoming more important factor in this process. This new measure provides a efficient method to evaluate the survivability of the network and design a network with high survivability. It is only a basic research in theory, How to use this measure with other factors such as network flows, cost, priority and reliability to set up a comprehensive and efficient model for MCNs is need further to study.

\section{REFERENCE}

[1] H. Kang, Study on survivability of tactical communication network, MSc thesis, 1994, XIDIAN university, Xi'an, P R China.
[2] Schroeder_MA, Newport_KT, Tactical network survivability through connectivity optimization, proceedings of IEEE Milcom 1987, pp.590-597

[3] Schroeder_MA, Newport_KT, Augmenting tactical communications networks to enhance survivability, proceedings of IEEE Milcom 1988, pp.507513

[4] Wu_L, Varshney_PK, On survivability measures for military networks, proceedings of IEEE Milcom 1990, pp.1120-1124

[5] L. Ying, A few of survivability measures of military communication network based on network connectivity, proceedings of the third military communication conference. 